\title{
Evaluation of Community Health Workers Performance at Home Based Newborn Assessment Supported by mHealth Tool in Rural Bangladesh
}

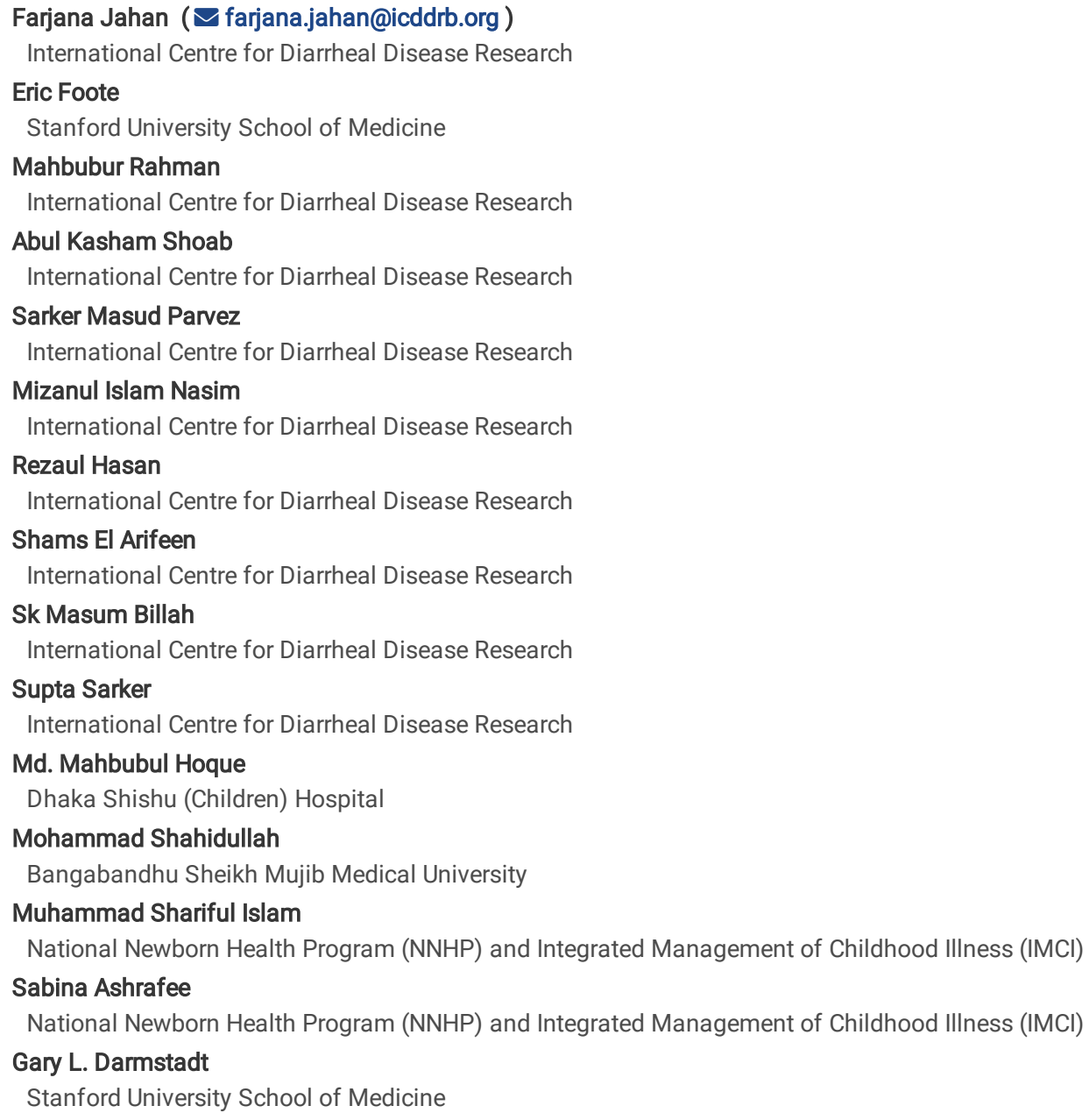




\section{Abstract}

Background: In low to middle-income countries where home births are common and neonatal postnatal care is limited, community health worker (CHW) home visits can extend the capability of health systems to reach vulnerable newborns in the postnatal period. CHW assessment of newborn danger signs supported by mHealth have potential to improve the quality of danger sign assessments and reduce CHW training requirements. We aim to estimate the validity (sensitivity, specificity, positive and negative predictive value) of CHW assessment of newborn infants aided by mHealth compared to physician assessment.

Methods: In this prospective study, ten CHWs received five days of theoretical and hands-on training on the physical assessment of newborns including ten danger signs. CHWs assessed 273 newborn infants for danger signs within 48 hours of birth and then consecutively for three days. A physician repeated $20 \%$ $(n=148)$ of the assessments conducted by $\mathrm{CHWs}$. Both $\mathrm{CHWs}$ and the physician evaluated newborns for ten danger signs and decided on referral. We used the physician's danger sign identification and referral decision as the gold standard to validate CHWs' identification of danger signs and referral decisions.

Results: The referrals made by the CHWs had high sensitivity (93.3\%), specificity (96.2\%), and almost perfect agreement (K=0.80) with the referrals made by the physician. CHW identification of all the danger signs except hypothermia showed moderate to high sensitivity (66.7-100\%) compared to physician assessment. All the danger signs assessments except hypothermia showed moderate to high positive predictive value (PPV) (50-100\%) and excellent negative predictive value (NPV) (99-100\%). Specificity was high (99-100\%) for all ten danger signs.

Conclusion: $\mathrm{CHW}$ identification of neonatal danger signs aided by mHealth showed moderate to high validity in comparison to physician assessment. mHealth platforms may reduce $\mathrm{CHW}$ training requirements and while maintaining quality $\mathrm{CHW}$ physical assessment performance extending the ability of health systems to provide neonatal postnatal care in low-resource communities.

Clinical Trial (CT) registration ID: NCT03933423

Name of the trial registry: clinicaltrials.gov

Clinical Trial (CT) registration Date: 01/05/2019

\section{Introduction}

Neonatal mortality remains a major challenge for achieving Sustainable Development Goal (SDG) 3 for good health and well-being in low and lower-middleincome countries (LMICs). [1] Over the 20 years, Bangladesh made progress in reducing under-5 mortality especially the post neonatal and child mortality. [2] But newborn mortality still a big challenge and contributes $61 \%$ of all under- 5 mortality. Three-quarters of neonatal deaths occur in the first week of life and nearly half occur in the first $24 \mathrm{~h}$, of which more than half occur at home. [2-5]

In Bangladesh, despite a reduction in neonatal mortality rate from 52 per 1000 live births in 1993-1994 to 30 per 1000 live births in $2017-18$, the decline is slow, and the rate remains well above the 12 per 1000 live birth target of SDG 3 by 2030. [6, 7] Approximately $50 \%$ of mothers in Bangladesh deliver at home, and a medical provider sees $52 \%$ of women and children within 48 hours of delivery. However, only $7 \%$ of home births are seen by a medical provider within 48 hours of birth. [8] To improve health and survival in the first month after birth, community-based interventions to link the community and healthcare system using home visits by $\mathrm{CHWs}$ have been explored extensively in LMIC settings. [9-17] CHW neonatal postnatal home visits have reduced neonatal mortality by about $30-60 \%$ among communities. [17-19]

During postnatal home visits, trained CHWs perform physical assessments to screen newborns for danger signs and those newborns with danger signs are referred to a healthcare center for treatment. [20-22] In order for CHWs to be able to make quality neonatal danger sign assessments, resource intensive trainings with a long duration were required varying from 10 days to 36 days, limiting countrywide scale up of CHW-led newborn postnatal programs. [14, 23 , 24]

Mobile applications and devices for public health activities (mHealth) have been explored to aid CHWs to provide maternal and child care in different settings across the world. [25-29] These tools can support in training, communication, data collection, decision-making, appropriate supervision, monitoring, management, and improve the performance and motivation of the CHWs. [30,31] In different settings, mHealth based programs modeled on the identification of mother and newborn at risk, delivery of care and follow-up demonstrated increases in outreach and feasibility. [32-35] In Bangladesh, a number of studies evaluated and demonstrated potential applications of mHealth in engaging $\mathrm{CHWs}$ in delivering maternal and newborn health intervention including message delivery for health promotion, identification of sick newborn and emergency referral. [36-40]

In this study, trained CHWs were evaluated for their ability to identify newborn danger signs during postnatal visits with the help of an mHealth tool with a built-in clinical algorithm.

We aimed to evaluate the effectiveness of our CHW training program to train CHWs to perform quality newborn danger sign assessments with the aid of mHealth in comparison to CHW training programs with longer training duration with a shorter training period and comprehensive training on improving CHWs' assessment of neonatal danger signs and the need for referral compared to physician assessment as the gold standard.

\section{Methods}

\section{Study design and population}


This prospective study was conducted within a cluster-randomized controlled intervention trial of home-based management of neonatal jaundice in Sakhipur Upazilla, Tangail, Bangladesh from September 2019 to March 2020 and October 2020 to April 2021. Sakhipur subdistrict of Dhaka division, Bangladesh is a rural agrarian community with a population of $~ 300,000$ people in 132 villages, approximately 80 kilometres from the capital city of Dhaka. A government hospital with 50 beds provides caesarean sections, vaginal birthing services, and emergency services. Poverty, crowding, unstable housing, food insecurity, and poor hygiene and sanitation are common. [41] About $82 \%$ of women in Dhaka division receive at least one antenatal care (ANC) visit from a trained medical provider, and $73 \%$ of women receive quality antenatal care. [8] Approximately $50 \%$ of mothers in the selected division (Dhaka) deliver at home, and a medical provider sees $52 \%$ of women and children within 48 hours of delivery. However, only $7 \%$ of home births are seen by a medical provider within 48 hours of birth. [8] If a neonatal hyperbilirubinemia case is diagnosed in Sakhipur and requires phototherapy, newborn infants are referred to a hospital that takes at least two hours to reach. [42]

For participant recruitment, we completed a listing of 650 pregnant mothers within 14-26 weeks of pregnancy from the Shakhipur sub-district along with Global Positioning System (GPS) locations of the households. We then formed 20 clusters, each comprised of 25-29 pregnant mothers residing in the closest geographical proximity. We eliminated households that did not form a cluster according to geographical proximity. We used Excel's rand function to assign clusters to intervention or control randomly. Randomization resulted in 10 clusters in each arm. All the live newborns of the enrolled pregnant mothers in the intervention clusters were included in the study for danger signs assessment and were assessed within 48 hours of birth.

\section{Recruitment of the CHWs}

Ten CHWs were recruited through a competitive recruitment process at the beginning of the project from the local community. We assigned one $\mathrm{CHW}$ to each of the ten intervention clusters. All the CHWs were female, ranging from 22 to 40 years of age and had a minimum of higher secondary education. A few of them had prior experience working in the community, and most of them had little knowledge of pregnant mother and newborn health care.

\section{Training of the CHWs}

We developed a training manual on prenatal educational sessions for mothers on antenatal care, neonatal jaundice, and home-based screening and management of neonatal jaundice in English and Bangla by adapting existing materials used in similar programs and standardizing the contents through face and content validation. All CHWs were given five days of training in two phases: perinatal care of the mother and management of neonatal jaundice (Table 1). Training included three days of theoretical and practical training at Sakhipur Upazilla Health Complex (UHC) and two days of bedside hands-on training at Dhaka Children Hospital; a tertiary level specialized hospital in Dhaka, Bangladesh. The research team evaluated their CHWs' pretest and posttest performance using a structured assessment tool. In addition, during hands-on training, the trainers observed each CHW assessing five newborns in the neonatal ward for danger signs. We conducted refresher training monthly. A weekly meeting was organized for experience sharing and review of study progress. 
Table 1

Curriculum contents of the Community Health Worker training

Theoretical and practical training topics
The scenario of maternal and child health in Bangladesh, neonatal jaundice, and responsibilities of community health workers

Pregnancy care and maternal danger signs

Delivery planning and safe delivery preparations

Newborn care

Prevention of maternal and neonatal infections

Breastfeeding and management of breastfeeding problems

Recognizing neonatal illness and common problems

Physical examination and evaluation of the newborn after birth

Postpartum services and the responsibilities of community health workers

Referral system of sick newborn and its components

Neonatal jaundice, types, and symptoms

Risk of developing neonatal jaundice and management

Management of neonatal jaundice in the community

Description of equipment and materials associated with jaundice management

Communication and counseling

Using an electronic handheld tablet and CommCare for case management

General examination of a neonate

Danger signs of the neonate

Infection prevention of the newborn

Neonatal jaundice signs, symptoms, and management

Breastfeeding and management of breastfeeding problems

\section{[INSERT Table 1]}

\section{Study procedure}

The CHW visited each newborn within 48 hours of birth and then daily for three consecutive days to assess for hyperbilirubinemia and to evaluate breastfeeding and institute phototherapy if indicated. Total bilirubin was measured using a transcutaneous bilimeter (Draeger JM-105). During CHW visits, if any danger signs was observed, CHWs referred newborns to an appropriate medical facility. Newborns eligible for home treatment were treated with LED phototherapy using Firefly ${ }^{\circledR}$ phototherapy system (MTTS Asia, Hanoi Vietnam), a portable double surface phototherapy device, for 36 hours. During the phototherapy period, CHWs assessed newborns daily for danger signs (Figure 1). [43]

\section{[INSERT Figure 1]}

A designated physician repeated the same assessments of newborn danger signs independently on the same day as the CHW assessment within 4 hours. The designated physician repeated $20 \%$ of the total assessments conducted by each CHW. On each day, the physician collected the list of the households the CHWs visited and conveniently picked households to repeat assessments. Physicians visited the households and repeated the CHWs assessments in newborns, both with and without danger signs. Physicians' identification of danger signs was used as the gold standard in determining the validity (sensitivity, specificity, and positive and negative predictive values) of CHWs' identification of danger signs of illness. For referral, the physician's decision on referral was considered as the final decision to prevent unnecessary referral.

\section{[INSERT Figure 2]}

\section{mHealth case management and decision support}

We developed an mHealth application (Figure 2) for CHWs using CommCare software program (Dimagi, Cambridge, USA) on an electronic handheld tablet to guide $\mathrm{CHWs}$ through newborn danger signs assessment. Successful use of the CommCare based mHealth applications by CHWs has been demonstrated previously. [44] The application contained pertinent information on the participants, including location, gestational age, blood group, and Rh type of pregnant women and their husbands.

The application developed on CommCare calculated an estimated delivery date based on the first day of the last menstrual period. [45] Gestational age was calculated by subtracting the birth date from the first day of the last menstrual period. The birth weight of the newborn was directly measured and also obtained by reviewing delivery records from the hospital. Birth weight of the newborns were measured by Seca weight scale (model: 383 1317009). Age of the 
baby in hours, was determined based on birth time reported by the mother or from records among hospital births. The mobile tablet guided the CHWs in 1) danger sign assessment of the newborn and mother, 2) breastfeeding assessment, and 3) neonatal jaundice case management by an algorithm based on AAP guidelines. [46] In every referral decision, the program provided a brief note describing the problem and emphasized the need for the referral for the $\mathrm{CHWs}$ and family members to understand. CHWs then facilitated the referral to Sakhipur sub-district hospital with the help of family members and the physician. CHWs communicated with the referral centre to ensure the referred newborn had been managed in the referral centre.

The application had data quality checks (answers constraints and requires questions to be answered before advancing) to improve the accuracy of CHW recommendations.

The neonatal danger signs used in this study were adopted from the Neonatal National Health Strategy which were validated previously in Bangladesh. [23, $47,48]$ The algorithm included ten danger signs for assessment. If a danger sign was present, the program prompted for referral. We added no void in 24 hours to the danger signs because we were going to be treating babies with phototherapy and did not want to treat babies who were already dehydrated. In case of hypothermia, CHWs advised mothers to warm up the babies and provide kangaroo mother care. After 30 minutes of warm-up, if the baby was still hypothermic, they referred the baby. If any of the following danger signs were present or if the newborn was $<2000$ grams or $<35$ weeks gestational age at birth, the newborn was referred to nearest UHC for treatment.

1. History of Convulsions- CHWs asked mother if the baby had a convulsion after birth

2. Hypothermia- if the axillary temperature measured by a digital thermometer was less than $35.5^{\circ} \mathrm{C}$ or $95.9^{\circ} \mathrm{F}$

3. Fever- if the axillary temperature measured by a digital thermometer was more than $37.5^{\circ} \mathrm{C}$ or $99.5^{\circ} \mathrm{F}$

4. Severe chest in drawing- $\mathrm{CHWs}$ checked if the newborn had severe chest indrawing

5. Tachypnea ( $>60$ breath/min on two consecutive readings, 30 minutes apart)

6. Umbilical redness extending to abdominal skin- $\mathrm{CHWs}$ checked if the baby has umbilical redness extending to abdominal skin

7. Movement only when stimulated or no movement at all-

8. No void within 24 hours- $\mathrm{CHWs}$ asked the mother if the baby had no void in last 24 hours

9. Jaundice of palms or soles- CHWs checked if the yellowness of body extended to palm or soles

10. Feeding difficulty- CHWs asked the mother if the baby had any feeding difficulty

\section{Statistical analysis}

The CHW-physician assessment pair was the unit of analysis. We examined the association between the assessment by physicians and assessment by the CHWs to identify individual danger signs. Sensitivity, specificity, positive predictive value (PPV), and negative predictive value (NPV) were calculated considering the assessment by the physician as the gold standard. Kappa statistics $(\mathrm{K})$ were calculated for determining agreement between physicians and CHWs. $\mathrm{K}<0$ was considered poor, $\mathrm{K}=0.0-0.20$ slight, $\mathrm{K}=0.21-0.40$ fair, $\mathrm{K}=0.41-0.60$ moderate, $\mathrm{K}=0.61-0.80$ substantial and $\mathrm{K}=0.81-1.00$ was considered almost perfect agreement [49]. Kappa tests were done considering alpha<0.05 as statistically significant. STATA 16 software package (Stata Corporation, College Station, TX, United States of America) was used for all the analysis.

\section{Ethical approval and consent to participate}

We took written informed consent from all the participants. Field researchers explained study objectives, activities, anonymity, and confidentiality to the participants and assured them that they could withdraw anytime. Any modifications to the protocol was communicated to the Institutional review boards to be approved for ethical clearance. All methods were performed in accordance to the trial protocol and guidelines. This study was approved by the Research Review Committee and the Ethical Review Committee of icddr,b, protocol \# 19004 and the Stanford University Institutional Review Board. The trial was registered at clinicaltrials.gov (NCT03933423) on 01/05/2019.

\section{Safety Review Plan}

An independent data safety monitoring committee (DSMB) was assembled in Bangladesh to monitor adverse events and to advise investigators with five respective members. The board was composed of two neonatologists, two epidemiologists, and one demographer. Stanford University institutional review board also reviewed the protocol prior to beginning the study. Any cases of acute bilirubin encephalopathy, exchange transfusion, or death during the trial was considered a serious adverse event. The investigators will report serious adverse events to the Data Safety Monitoring Board within 24 hours of the event. The Data Safety Monitoring Board was responsible for evaluating the event and determining the next steps. To ensure project safety, study staff and a project research physician were always present in the field site.

\section{Results}

\section{Characteristics of CHWs}

The mean age of the CHWs was $28.1( \pm 3.2)$ years. About three quarters $(71 \%)$ of the $\mathrm{CHWs}$ had completed graduation, and no $\mathrm{CHWs}$ had any previous training on identifying newborn danger signs.

\section{Assessments}


A total of 273 newborns born between November 2019 to March 2020 and October 2020 to May 2021 were assessed by the CHWs. CHWs completed a total of 740 assessments. Among 273 newborns, 27 (9.8\%) newborns were referred or started phototherapy on the first day of assessment, and 28 (10.2\%) were referred or started phototherapy on the second day of assessment.

\section{Characteristics of the newborns}

Out of the 148 newborns assessed by both a CHW and the physician, $45 \%$ were female (Table 2). Eight percent of the neonates were born before 35 weeks gestational age, $16 \%$ were born between $35-37$ weeks of gestation, and $41 \%$ were born at home. The mean birth weight was 2658 ( \pm 375 ) grams and only nine $(6 \%)$ of the newborns had low birth weight (<2500gm) (Table 2). There were no babies with birth weight less than 2000 gram. The average hour of life of the newborns during first assessments was 50 (SD 39) hours.

Table 2

Characteristics of newborn infants included in the $\mathrm{CHW}$ assessment validation study in Shakhipur, Bangladesh (2019-2020)

\begin{tabular}{|lll|}
\hline Characteristics & Frequency (n=148) & Percentage (\%) \\
\hline Sex & $67 / 148$ & 45 \\
Female & $81 / 148$ & 55 \\
\hline Gestational age & & \\
\hline$<35$ weeks & & 8 \\
\hline $35-37$ weeks & $11 / 148$ & 16 \\
\hline$\geq 37$ weeks & $25 / 148$ & 76 \\
\hline Birth place & $112 / 148$ & 6 \\
\hline UHC & & 3 \\
\hline District/ Medical college hospital & $5 / 148$ & 48 \\
\hline Private hospital & $71 / 148$ & 1 \\
\hline NGO clinic & $2 / 148$ & 41 \\
\hline Home & $61 / 148$ & \\
\hline Mode of delivery & & 57 \\
\hline Normal vaginal delivery & $118 \pm 51$ & \\
\hline Cesarean section & $85 / 148$ & \\
\hline Birth weight (in grams), mean (SD) & $2658(375)$ & \\
\hline Birthweight & 139 & \\
\hline$\geq 2500$ gm & $93 / 148$ & \\
\hline $2000-2499$ gm & & \\
\hline Hour of life during assessments & $($ Mean, SD) & \\
\hline Assessment 1 & $50 \pm 39$ & \\
\hline Assessment 2 & & \\
\hline Assessment 3 & & \\
\hline
\end{tabular}

\section{[INSERT Table 2]}

\section{Assessment of newborn danger signs}

During 148 CHWs' assessments, 16 (11\%) newborns had a single danger sign, one had two danger signs, and two newborns had four danger signs. The danger signs found most frequently during $\mathrm{CHW}$ assessments were no void in 24 hours (4.7\%), newborns with yellow palms and soles (2.7\%), and difficulty feeding (2\%) (Table 3). Hypothermia and hyperthermia were reported among $2 \%$ and $1.4 \%$ of newborns, respectively. Convulsions were found in $1.4 \%$ of newborns, and they moved only while being stimulated. Severe chest in-drawing and umbilical redness extending to the abdominal skin were observed in $0.7 \%$ of newborns. CHWs did not report any case of tachypnea, and sensitivity and specificity of tachypnea could not be reported. 
Table 3

Distribution of danger signs by gestational age and birthweight of newborn

\begin{tabular}{|c|c|c|c|c|c|c|}
\hline \multirow[t]{3}{*}{ Danger signs } & Total Frequency n (\%) & \multicolumn{3}{|c|}{ Gestational age n (\%) } & \multicolumn{2}{|c|}{ Birth weight n (\%) } \\
\hline & & $<35$ weeks & 35-37 weeks & $>37$ weeks & $2000-2499 \mathrm{gm}$ & $>=2500 \mathrm{gm}$ \\
\hline & & n (11) & n (25) & n (112) & $\mathrm{n}(9)$ & n (139) \\
\hline
\end{tabular}

\begin{tabular}{|lllllll|}
\hline Convulsion & $2(1.4)$ & $0(0)$ & $0(0)$ & $2(100)$ & $0(0)$ & $2(100)$ \\
\hline Hypothermia & $3(2.0)$ & $1(33.3)$ & $1(33.3)$ & $1(33.3)$ & $0(0)$ & $3(100)$ \\
\hline Hyperthermia & $2(1.4)$ & $0(0)$ & $0(0)$ & $2(100)$ & $0(0)$ & $2(100)$ \\
\hline Severe chest-indrawing & $1(0.7)$ & $0(0)$ & $0(0)$ & $1(100)$ & $0(0)$ & $1(100)$ \\
\hline Tachypnea & $0(0)$ & $0(0)$ & $0(0)$ & $0(0)$ & $0(0)$ & $0(0)$ \\
\hline Umbilical redness extending to the skin & $1(0.7)$ & $1(100)$ & $0(0)$ & $0(0)$ & $1(50)$ & $0(0)$ \\
\hline Only moves when stimulated & $2(1.4)$ & $1(50)$ & $0(0)$ & $1(100)$ \\
\hline No void in 24 hours & $7(4.7)$ & $2(29)$ & $1(14)$ & $4(57)$ & $0(0)$ & $0(100)$ \\
\hline Yellow palm or soles & $4(2.7)$ & $0(0)$ & $0(0)$ & $4(100)$ & $0(0)$ & $4(100)$ \\
\hline Feeding difficulty & $3(2.0)$ & $1(33)$ & $0(0)$ & $2(67)$ & $0(0)$ \\
\hline
\end{tabular}

\section{[INSERT Table 3]}

Convulsion, severe chest in drawing, umbilical redness extending to abdominal skin and no void in 24 hours had $100 \%$ sensitivity and almost perfect agreement $(K=0.83-1.00)$. Feeding difficulty and no movement or only moves when stimulated had moderate to high sensitivity and positive predictive value resulting in almost perfect agreement $(\mathrm{K}=0.85,0.83)$. Hyperthermia and yellow palm or soles had $100 \%$ and $75 \%$ sensitivity while positive predictive values were $50 \%$ and $75 \%$, respectively resulting in substantial agreement $(K=0.66,0.74)$. On the other hand, hypothermia had $50 \%$ sensitivity and $33.3 \%$ positive predictive value, and the agreement was fair $(K=0.39)$. Specificity and negative predictive value were above $99 \%$ for all the danger signs.

According to the danger signs, $12.8 \%$ of the newborns were referred by CHWs. The sensitivity of referral was $93.3 \%$, and specificity was $96.2 \%$. Positive predictive value of referral was $73.7 \%$, and negative predictive value was $99.2 \%$. Agreement between $\mathrm{CHW}$ referral and physician referral was almost perfect (K=0.80) (Table 4). 
Table 4

Frequency (\%) and validity of clinical signs observed, referral decision and Kappa statistic for agreement between assessments by CHWs and physician gold s

\begin{tabular}{|c|c|c|c|c|c|c|c|c|c|c|c|}
\hline $\begin{array}{l}\text { Newborn } \\
\text { danger signs } \\
\text { assessed }\end{array}$ & $\begin{array}{l}\text { Number of } \\
\text { children had } \\
\text { the } \\
\text { sign/symptoms } \\
\text { (identified by } \\
\text { physician) } \\
\mathrm{n}(\%)\end{array}$ & $\begin{array}{l}\text { Number of } \\
\text { children did not } \\
\text { have the } \\
\text { sign/symptoms } \\
\text { (identified by } \\
\text { physician) } \\
\mathrm{n}(\%)\end{array}$ & $\begin{array}{l}\text { True } \\
\text { negative }\end{array}$ & $\begin{array}{l}\text { False } \\
\text { negative }\end{array}$ & $\begin{array}{l}\text { False } \\
\text { positive }\end{array}$ & $\begin{array}{l}\text { True } \\
\text { positive }\end{array}$ & $\begin{array}{l}\text { Sensitivity } \\
\%(\mathrm{Cl})\end{array}$ & $\begin{array}{l}\text { Specificity } \\
\%(\mathrm{Cl})\end{array}$ & $\begin{array}{l}\text { PPV } \\
\% \\
\text { (Cl) }\end{array}$ & $\begin{array}{l}\text { NPV } \\
\% \\
\text { (Cl) }\end{array}$ & $\mathrm{K}$ \\
\hline $\begin{array}{l}\text { Convulsion } \\
(\mathrm{N}=148)\end{array}$ & $2(1.4)$ & 146 (98.7) & 146 & 0 & 0 & 2 & $\begin{array}{l}100.0 \\
(15.8-100)\end{array}$ & $\begin{array}{l}100.0 \\
(97.5-100)\end{array}$ & $\begin{array}{l}100.0 \\
(15.8- \\
100)\end{array}$ & $\begin{array}{l}100.0 \\
(97.5- \\
100)\end{array}$ & 1.00 \\
\hline $\begin{array}{l}\text { Hypothermia } \\
(\mathrm{N}=148)\end{array}$ & $1(0.7)$ & 147 (99.3) & 144 & 1 & 2 & 1 & $\begin{array}{l}50.0 \\
(1.3-98.7)\end{array}$ & $\begin{array}{l}98.6 \\
(95.1- \\
99.8)\end{array}$ & $\begin{array}{l}33.3 \\
(0.8- \\
90.6)\end{array}$ & $\begin{array}{l}99.3 \\
(96.2- \\
100)\end{array}$ & 0.39 \\
\hline $\begin{array}{l}\text { Hyperthermia } \\
(\mathrm{N}=148)\end{array}$ & $1(0.7)$ & 147 (99.3) & 146 & 0 & 1 & 1 & $\begin{array}{l}100 \\
(2.5-100)\end{array}$ & $\begin{array}{l}99.3 \\
(96.3-100)\end{array}$ & $\begin{array}{l}50.0 \\
\left(1.3^{-}\right. \\
98.7)\end{array}$ & $\begin{array}{l}100 \\
(97.5- \\
100)\end{array}$ & 0.66 \\
\hline $\begin{array}{l}\text { SevereFechest } \\
\text { in-drawing } \\
(\mathrm{N}=148)\end{array}$ & $1(0.7)$ & 147 (99.3) & 147 & 0 & 0 & 1 & $\begin{array}{l}100 \\
(2.5-100)\end{array}$ & $\begin{array}{l}100 \\
(97.5-100)\end{array}$ & $\begin{array}{l}100 \\
(2.5- \\
100)\end{array}$ & $\begin{array}{l}100 \\
(97.5- \\
100)\end{array}$ & 1.00 \\
\hline $\begin{array}{l}\text { Tachypnea } \\
(\mathrm{N}=148)\end{array}$ & $1(0.7)$ & 147 (99.3) & 147 & 0 & 1 & 0 & $\left.{ }^{*}\right)$ one-sidec & $97.5 \%$ confi & ence int & rval & \\
\hline $\begin{array}{l}\text { Baby has } \\
\text { umbilical } \\
\text { redness } \\
\text { extending to } \\
\text { abdominal } \\
\text { skin }(\mathrm{N}=148)\end{array}$ & $1(0.7)$ & 147 (99.3) & 147 & 0 & 0 & 1 & $\begin{array}{l}100.0 \\
(2.5-100)\end{array}$ & $\begin{array}{l}100.0 \\
(97.5-100)\end{array}$ & $\begin{array}{l}100.0 \\
(2.5- \\
100)\end{array}$ & $\begin{array}{l}100.0 \\
(97.5- \\
100)\end{array}$ & 1.00 \\
\hline $\begin{array}{l}\text { Only moves } \\
\text { when } \\
\text { stimulated } \\
(\mathrm{N}=148)\end{array}$ & $3(2)$ & 145 (98) & 145 & 1 & 0 & 2 & $\begin{array}{l}66.7 \\
(9.4-99.2)\end{array}$ & $\begin{array}{l}100 \\
(97.5-100)\end{array}$ & $\begin{array}{l}100 \\
(15.8- \\
100)\end{array}$ & $\begin{array}{l}99.3 \\
(96.2- \\
100)\end{array}$ & 0.80 \\
\hline $\begin{array}{l}\text { Newborn had } \\
\text { no void (urine) } \\
\text { in } 24 \text { hours } \\
(\mathrm{N}=148)\end{array}$ & $5(3.4)$ & $143(96.6)$ & 141 & 0 & 2 & 5 & $\begin{array}{l}100.0 \\
(47.8-100)\end{array}$ & $\begin{array}{l}98.6 \\
(95.0- \\
99.8)\end{array}$ & $\begin{array}{l}71.4 \\
(29.0- \\
96.3)\end{array}$ & $\begin{array}{l}100.0 \\
(97.4- \\
100)\end{array}$ & 0.83 \\
\hline $\begin{array}{l}\text { Newborn had } \\
\text { yellow palm } \\
\text { or soles of } \\
\text { feet }(N=148)\end{array}$ & $4(2.7)$ & 144 (97.3) & 143 & 1 & 1 & 3 & $\begin{array}{l}75.0 \\
(19.4- \\
99.4)\end{array}$ & $\begin{array}{l}99.3 \\
(96.2-100)\end{array}$ & $\begin{array}{l}75.0 \\
(19.4- \\
99.4)\end{array}$ & $\begin{array}{l}99.3 \\
(96.2- \\
100)\end{array}$ & 0.74 \\
\hline $\begin{array}{l}\text { Baby having } \\
\text { difficulty } \\
\text { feeding } \\
(\mathrm{N}=148)\end{array}$ & $4(2.7)$ & 144 (97.3) & 144 & 1 & 0 & 3 & $\begin{array}{l}75.0 \\
(19.4- \\
99.4)\end{array}$ & $\begin{array}{l}100 \\
(97.5-100)\end{array}$ & $\begin{array}{l}75.0 \\
(29.4- \\
100)\end{array}$ & $\begin{array}{l}99.3 \\
(96.2- \\
100)\end{array}$ & 0.85 \\
\hline $\begin{array}{l}\text { CHW decision } \\
\text { on } \\
\text { assessment } \\
\text { Baby has } \\
\text { danger signs } \\
\text { and need } \\
\text { referral } \\
(\mathrm{N}=148)\end{array}$ & 15 (10.1) & 133 (89.9) & 128 & 1 & 5 & 14 & $\begin{array}{l}93.3 \\
(68.1- \\
99.8)\end{array}$ & $\begin{array}{l}96.2 \\
(91.4- \\
98.8)\end{array}$ & $\begin{array}{l}73.7 \\
(48.8- \\
90.9)\end{array}$ & $\begin{array}{l}99.2 \\
(95.8- \\
100)\end{array}$ & 0.80 \\
\hline
\end{tabular}

${ }^{*}$ ) one-sided, $97.5 \%$ confidence interval

- SE (Standard Error), TN (True Negative), FN (False negative), (FP) False Positive, TP (True Positive)

- Some validity measures could not be calculated for signs with no cases identified by CHW

Physician's assessment prevented five (25\%) referrals that were made by CHWs. After being referred 15 (100\%) of the newborns received management either at government hospital or private clinic. After referral, 13 (86.6\%) newborns were admitted to the hospital, and two newborns were checked by pediatricians in a chamber.

\section{[INSERT Table 4]}


This study was conducted to evaluate the validity of trained CHWs compared to a physician gold standard in assessing danger signs of newborns in a rural, low-resource setting in Bangladesh with the assistance of an algorithm run through a mobile device. Study results showed that, $\mathrm{CHW}$ led home visits led to $12.8 \%$ referral with a successful referral rate of $100 \%$. This implies a high acceptance of CHWs based assessment at community level. However, this evaluation has its own limitation. Physician's assessment might influence the success rate of referral, parents might be more convinced as the physician visited and assessed their newborn. Further research would be required to evaluate the acceptance of CHW's referral if physician does not intervene. Similar to our findings, in other contexts mHealth based home visits have been shown to be effective in improving care seeking behavior in neonatal period. [50-53] Results showed that the referrals made by the $\mathrm{CHWs}$ had high sensitivity $(93.3 \%)$, specificity $(96.2 \%)$, and almost perfect agreement $(\mathrm{K}=0.80)$ with the referrals made by the physician. Mobile health-based assessment showed high level of adequacy in identifying newborn with danger signs. This finding corresponds to the previous validation study conducted in Bangladesh. [14, 22, 23]

The CHW training was relatively short compared to other studies (5 days, as opposed to 36 days of training in the Mirzapur study [22] and 42 days of training in the Sylhet study. [14] mHealth may have contributed to the ability of $\mathrm{CHWs}$ to perform quality danger sign assessments despite a shorter training program.

In a study conducted among 288 neonates in rural Sylhet, Bangladesh, CHWs identified and referred infants with danger signs categorized as very severe disease with a sensitivity of $91 \%$ ( $K=0.847)$ and infants with possible very severe disease with a sensitivity of $81 \%$ ( $K=0.783$ ) compared to physician assessment. [14] The frequencies of both very severe disease and possible very severe disease were $25.7 \%$ and $20.5 \%$, respectively, and there were equal numbers of well and sick newborns in the study. Another study conducted in Mirzapur, Bangladesh among 395 infants showed that CHWs identified infants with very severe disease and possible very severe disease with $72.7 \%(K=0.63)$ and $33.3 \%(K=0.33)$ sensitivity, respectively compared to physician gold standard. [22] In this study, very severe disease was defined, as presence of one or more signs among 11 danger signs: convulsions, unconscious, fast breathing, severe chest-indrawing, fever, hypothermia, severe skin pustules, umbilical redness extending to skin, weak, abnormal or absent cry, lethargic or less movement, and feeding difficulty. [22] Very severe disease was found among $2.8 \%$ of newborns, and possible very severe disease was found among $6.8 \%$ of the newborns. CHWs referred newborns identified with very severe disease or possible very severe disease in both studies. The frequency of referral in our current study was $12.8 \%$ while in these two studies, it was $9.6 \%$ and $26 \%$, respectively.

Another study by Biswas et al. found that among babies 2-59 months of age, frontline community workers (Auxiliary Nurse Midwives, Anganwadi Workers) correctly assessed $33.6 \%$ of the children for all four danger signs following integrated management of neonatal and childhood illness (IMNCI) - which is lower than our study. [54] In a study conducted in Nepal among 1,448 young infants, there was substantial agreement (K>0.75) between home assessments conducted by female community health volunteers (FCHV) with basic training compared to home assessments conducted by highly trained facility-based community health workers (FBCHW). [24] A recent study conducted in Ghana compared community volunteer assessments with non-physician trained supervisor observation and found high validity (79.5\% sensitivity, $100 \%$ specificity, and $96.6 \%$ agreement). [21]

The positive predictive value $(73.7 \%, 48.4-90.9 \% \mathrm{Cl})$ of referral in our study indicates that about three-fourths of the newborns needing referral-level care were correctly identified. It also indicates that nearly one-fourth of the neonates were diagnosed inaccurately, which may have caused unnecessary referrals. However, subsequent physician assessment prevented five $(25 \%)$ such referrals. NPV was high $(99.2 \%, 95.8-100 \% \mathrm{Cl})$ indicating there was less than one percent chance of a sick newborn not being referred. These findings are on par with similar studies mentioned above.

All the danger signs assessments except hypothermia had moderate to high positive predictive value (50-100\%) and excellent negative predictive value (98$100 \%)$. As this study was conducted in the general population, it implies CHWs' assessment can identify and refer a good number of diseased newborns and simultaneously reduce unnecessary exposure of newborns to hospitals.

The low sensitivity and agreement of hypothermia can be a result of multiple limitations of this study. Once any newborn was found hypothermic, CHWs advised mothers to warm up the babies providing kangaroo mother care and warm clothes. The physician assessed these newborns after 4 hours, and by this time, the babies became normothermic after warm-up procedures.

As the physician visited each household after the CHW visit, breathing rate may have changed by the time the physician visited the household (timing bias). Despite efforts to shorten the time gap between CHW visits and physician visits, a time lag is inevitable in this type of community-based study.

The prevalence of each of the danger signs in this study corresponded to other studies. Among the danger signs, no void in 24 hours was the most prevalent (4.7\%) and had high sensitivity (100\%). Hyperthermia, convulsion, and umbilical redness had higher sensitivity (100\%) than other validation studies (0-50\%) [22]. Prevalence of yellow palm or soles was $2.7 \%$ with sensitivity of $75 \%$ which corresponds to the neonatal jaundice identification validation study in Karachi, Pakistan (83.3\%) [55] but is higher than validation study in Bangladesh (21.4\%). [14, 22, 23, 56] The core study on neonatal jaundice, where CHWs measured the transcutaneous bilirubin to screen for neonatal jaundice may have influenced the CHWs ability to correctly identify this danger sign. Identification of this sign is also reliant on visual inspection and more objective than many other signs. [57] However, in a study conducted in Karachi, Pakistan, assessment of jaundice using visual inspection by primary health care workers had higher sensitivity than physicians' inspection ( $83.3 \%$ vs $51.4 \%$ ) but lower specificity (50.5\% vs $90.7 \%)$. [55]

Feeding difficulty was one of the most prevalent danger signs (2\%), and had high sensitivity (75\%) which corresponds to other studies, which ranged from $45-$ $100 \%$. $[14,23,56]$ The CHWs assessed breastfeeding and provided necessary instruction to the mother to feed the baby after the assessment. Though the CHWs assessed breastfeeding, the male physician did not because of cultural sensitivity.

Due to an inadequate number of newborns with tachypnea, we could not report the sensitivity and specificity of tachypnea in comparison to physician assessment. 
One of the limitations of this study is the sample size was small compared to other studies discussed above. It has been suggested that to optimize validation study design, healthy and sick newborn infants should be equal in number. [14] However, in our study only 19 newborns (12.8\%) were referred by the CHWs, and $15(10.1 \%)$ were referred by the physician. Our study tried to look at the CHWs' performance in the case of premature babies ( $\mathrm{n}=26)$. However, we did not have any newborn with birth weight <2000 gram, so we could not comment on CHWs' performance on very low birth weight babies. Future studies can focus on larger group validation in very low birth weight babies.

\section{Conclusion}

m-Health based, CHW-led assessment of newborns can be potential strategy to reduce neonatal mortality and morbidity in underdeveloped and developing country settings. This study corroborates that mobile-health based assessment after five days of training and skill development can effectively extend the capabilities of community health workers to reach the most vulnerable newborns in rural areas that are less likely to receive postnatal care. Increasing access to quality postnatal care has been shown to reduce neonatal mortality. mHealth application training can potentially eliminate the need of long course extensive training of CHWs in providing new born care and barriers towards scaling up. CHWs can provide basic healthcare at the doorsteps in resource-poor communities efficiently if monitored and supervised well.

\section{Abbreviations}

\section{LMIC}

Low- and Middle-Income Countries

CHW

Community Health Worker

ANC

Antenatal Care

PNC

Postnatal care

AAP

American Academy of Pediatrics

UHC

Upazilla Health Complex (Sub-district hospital)

NGO

Non-Government Organization

TcB

Transcutaneous bilirubin

\section{Declarations}

\section{Ethical approval and consent to participate}

We took informed written consent from all the participants. Field researchers explained study objectives, activities, anonymity, and confidentiality to the participants and assured them that they could withdraw anytime. This study was approved by the Ethical Review Committee and the Research Review Committee of icddr,b and the Stanford University Institutional Review Board. All methods were performed in accordance to the trial protocol and guidelines. The trial was registered for clinical trial (NCT03933423) on 01/05/2019.

\section{Consent for publication}

Not applicable.

\section{Availability of data and materials}

Institutional Review Board approval was obtained for public sharing and presentation of data on a group level only. To maintain participants' anonymity and confidentiality, the data set generated during the study will not be shared.

\section{Competing interests}

The authors declare that they have no competing interests.

\section{Funding}

The study was funded by the Grand Challenges Canada (SB-POC-1810-20535).

\section{Author's contributions}

FJ was a major contributor in implementing the study, analyzing and interpreting data, and writing the manuscript. EF, MR, AKS, SMP, MIN, RH and SS were substantial contributors in study design, implementation, and acquisition of data. MR was the Principal Investigator and overall implementer of the study. SEA, 
SMB, MMH, MS, MSI and SA participated in the study design and provided feedback on the manuscript. GLD was a major contributor in writing the manuscript and provided final approval of the version to be submitted. All authors have read and approved the final manuscript.

\section{Acknowledgements}

icddr,b acknowledges with gratitude the commitment of Grand Challenges Canada to its research supports. icddr,b is also grateful to the Governments of Bangladesh, Canada, Sweden and the UK for providing core/unrestricted support. The study team is grateful to the pregnant mother and grandparents who participated in the study and field workers and supervisors who implemented the interventions. We acknowledge the community health workers who were the key implementers of this study and the physician and nurses of Shakhipur Health Complex who provided continuous support in management of the referred newborn.

\section{References}

1. Lee BX, Kjaerulf F, Turner S, Cohen L, Donnelly PD, Muggah R, Davis R, Realini A, Kieselbach B, MacGregor LS: Transforming our world: implementing the 2030 agenda through sustainable development goal indicators. Journal of public health policy 2016, 37(1):13-31.

2. Minnery M, Firth S, Hodge A, Jimenez-Soto E: Neonatal mortality and inequalities in Bangladesh: Differential progress and sub-national developments. Maternal and child health journal 2015, 19(9):2038-2047.

3. Lozano R, Naghavi M, Foreman K, Lim S, Shibuya K, Aboyans V, Abraham J, Adair T, Aggarwal R, Ahn SY: Global and regional mortality from 235 causes of death for 20 age groups in 1990 and 2010: a systematic analysis for the Global Burden of Disease Study 2010. The lancet 2012, 380(9859):20952128 .

4. Lozano R, Wang H, Foreman KJ, Rajaratnam JK, Naghavi M, Marcus JR, Dwyer-Lindgren L, Lofgren KT, Phillips D, Atkinson C: Progress towards Millennium Development Goals 4 and 5 on matemal and child mortality: an updated systematic analysis. The lancet 2011, 378(9797):1139-1165.

5. WHO U: Every newborn: an action plan to end preventable deaths. Geneva: World Health Organization 2014.

6. Ahsan KZ JK, Islam S, Al-Sabir A, Chakraborty N: Bangladesh Demographic and Health Survey 2017-18: Key Indicators Report. 2019.

7. Ferri N: United nations general assembly. International Journal of Marine and Coastal Law 2010, 25:271-287.

8. National Institute of Population Research and Training MEaFWD, Ministry of Health and Family Welfare: Bangladesh Demographic and Health Survey, 2017-18. In.; 2018.

9. Chanani S, Waingankar A, Shah More N, Pantvaidya S, Fernandez A, Jayaraman A: Participation of pregnant women in a community-based nutrition program in Mumbai's informal settlements: Effect on exclusive breastfeeding practices. PLoS ONE 2018, 13:1-17.

10. Memon ZA, Khan GN, Soofi SB, Baig IY, Bhutta ZA: Impact of a community-based perinatal and newborn preventive care package on perinatal and neonatal mortality in a remote mountainous district in Northern Pakistan. BMC Pregnancy and Childbirth 2015, 15:1-9.

11. Perez K, Patterson J, Hinshaw J, Escobar C, Parajon D, Parajon L, Bose C: Essential Care for Every Baby: Improving compliance with newborn care practices in rural Nicaragua. BMC Pregnancy and Childbirth 2018, 18:1-9.

12. Namazzi G, Okuga M, Tetui M, Kananura RM, Kakaire A, Namutamba S, Mutebi A, Kiwanuka SN, Ekirapa-Kiracho E, Waiswa P: Working with community health workers to improve maternal and newborn health outcomes: Implementation and scale-up lessons from eastern Uganda. Global Health Action $2017,10$.

13. Sitrin D, Guenther T, Waiswa P, Namutamba S, Namazzi G, Sharma S, Ashish KC, Rubayet S, Bhadra S, Ligowe R et al: Improving newborn care practices through home visits: Lessons from Malawi, Nepal, Bangladesh, and Uganda. Global Health Action 2015, 8.

14. Baqui AH, Arifeen SE, Rosen HE, Mannan I, Rahman SM, Al-Mahmud AB, Hossain D, Das MK, Begum N, Ahmed S et al: Community-based validation of assessment of newborn illnesses by trained community health workers in Sylhet district of Bangladesh. Tropical Medicine and International Health 2009 , 14:1448-1456.

15. Aboubaker S, Qazi S, Wolfheim C, Oyegoke A, Bahl R: Community health workers: A crucial role in newborn health care and survival. Journal of Global Health 2014, 4:1-5.

16. Tripathi A, Kabra SK, Sachdev HPS, Lodha R: Home visits by community health workers to improve identification of serious illness and care seeking in newborns and young infants from low- and middle-income countries. Journal of Perinatology 2016, 36:S73-S81.

17. Baqui AH, El-Arifeen S, Darmstadt GL, Ahmed S, Williams EK, Seraji HR, Mannan I, Rahman SM, Shah R, Saha SK et al: Effect of community-based newborn-care intervention package implemented through two service-delivery strategies in Sylhet district, Bangladesh: a cluster-randomised controlled trial. The Lancet 2008, 371:1936-1944

18. Bang AT, Bang RA, Baitule SB, Reddy MH, Deshmukh MD: Lancet Field Trial. 2005, 354:1-7

19. Bhutta ZA, Memon ZA, Soofi S, Salat MS, Cousens S, Martines J: Implementing community-based perinatal care: Results from a pilot study in rural Pakistan. Bulletin of the World Health Organization 2008, 86:452-459.

20. (WHO) WHO: Postnatal care of the mother and newborn 2013. World Health Organization 2013:1-72.

21. Manu AA, Ten Asbroek A, Soremekun S, Gyan T, Weobong B, Tawiah-Agyemang C, Danso S, Amenga-Etego S, Owusu-Agyei S, Hill Z et al: Evaluating the implementation of community volunteer assessment and referral of sick babies: Lessons learned from the Ghana Newhints home visits cluster randomized controlled trial. Health Policy and Planning 2014, 29:ii114-ii127.

22. Darmstadt GL, Baqui AH, Choi Y, Bari S, Rahman SM, Mannan I, Nawshad Uddin Ahmed ASM, Saha SK, Rahman R, Chang S et al: Validation of community health workers' assessment of neonatal illness in rural Bangladesh. Bulletin of the World Health Organization 2009, 87:12-19. 
23. Darmstadt GL, Baqui AH, Choi Y, Bari S, Rahman SM, Mannan I, Ahmed ANU, Saha SK, Seraji HR, Rahman R: Validation of a clinical algorithm to identify neonates with severe illness during routine household visits in rural Bangladesh. Archives of disease in childhood 2011, 96(12):1140-1146.

24. Khanal S, Jaganath Sharma VSG, Dawson P, Houston R, Khadka N, Yengden B: Community health workers can identify and manage possible infections in neonates and young infants: MINI-a model from Nepal. Journal of health, population, and nutrition 2011, 29(3):255.

25. et al: Development and evaluation of a mobile application for case management of small and sick newborns in Bangladesh. BMC Medical Informatics and Decision Making 2019, 19.

26. Ilozumba O, Dieleman M, Kraamwinkel N, Van Belle S, Chaudoury M, Broerse JEW: "I am not telling. the mobile is telling": Factors influencing the outcomes of a community health worker mHealth intervention in India. PLOS ONE 2018, 13:1-23.

27. Källander K, Tibenderana JK, Akpogheneta OJ, Strachan DL, Hill Z, Asbroek AHAT, Conteh L, Kirkwood BR, Meek SR: Mobile health (mhealth) approaches and lessons for increased performance and retention of community health workers in lowand middle-income countries: A review. Journal of Medical Internet Research 2013, 15

28. Agarwal S, Perry HB, Long LA, Labrique AB: Evidence on feasibility and effective use of mHealth strategies by frontline health workers in developing countries: Systematic review. Tropical Medicine and International Health 2015, 20:1003-1014.

29. Praveen D, Patel A, Raghu A, Clifford GD, Maulik PK, Abdul AM, Mogulluru K, Tarassenko L, MacMahon S, Peiris D: SMARTHealth India: development and field evaluation of a mobile clinical decision support system for cardiovascular diseases in rural India. JMIR mHealth and uHealth 2014, 2(4):e3568.

30. Winters N, Langer L, Geniets A: Scoping review assessing the evidence used to support the adoption of mobile health (mHealth) technologies for the education and training of community health workers (CHWs) in low-income and middle-income countries. BMJ open 2018, 8(7):e019827.

31. Svoronos T, Mjungu P, Dhadialla R, Luk R, Zue C, Jackson J, Lesh N: CommCare: Automated quality improvement to strengthen community-based health. Weston: D-Tree International 2010

32. Prinja S, Nimesh R, Gupta A, Bahuguna P, Gupta M, Thakur JS: Impact of $m$-health application used by community health volunteers on improving utilisation of maternal, new-born and child health care services in a rural area of Uttar Pradesh, India. Tropical Medicine \& International Health 2017, 22(7):895-907.

33. Prinja S, Nimesh R, Gupta A, Bahuguna P, Thakur JS, Gupta M, Singh T: Impact assessment and cost-effectiveness of m-health application used by community health workers for maternal, newborn and child health care services in rural Uttar Pradesh, India: a study protocol. Global health action 2016, 9(1):31473.

34. Schoen J, Mallett JW, Grossman-Kahn R, Brentani A, Kaselitz E, Heisler M: Perspectives and experiences of community health workers in Brazilian primary care centers using m-health tools in home visits with community members. Human resources for health 2017, 15(1):1-10.

35. Schuttner L, Sindano N, Theis M, Zue C, Joseph J, Chilengi R, Chi BH, Stringer JS, Chintu N: A mobile phone-based, community health worker program for referral, follow-up, and service outreach in rural Zambia: outcomes and overview. TELEMEDICINE and e-HEALTH 2014, 20(8):721-728.

36. Alam MZ: mHealth in Bangladesh: current status and future development. The International Technology Management Review 2018, 7(2):112-124.

37. Crehan C, Kesler E, Nambiar B, Dube Q, Lufesi N, Giaccone M, Normand C, Azad K, Heys M: The NeoTree application: developing an integrated mHealth solution to improve quality of newborn care and survival in a district hospital in Malawi. BMJ global health 2019, 4(1):e000860.

38. Goel S, Bhatnagar N, Sharma D, Singh A: Bridging the human resource gap in primary health care delivery systems of developing countries with mhealth: narrative literature review. JMIR mHealth and uHealth 2013, 1(2):e2688.

39. Islam MN, Karim MM, Inan TT, Islam AN: Investigating usability of mobile health applications in Bangladesh. BMC medical informatics and decision making 2020, 20(1):1-13.

40. Jo Y, Labrique AB, Lefevre AE, Mehl G, Pfaff T, Walker N, Friberg IK: Using the lives saved tool (LiST) to model mHealth impact on neonatal survival in resource-limited settings. PloS one 2014, 9(7):e102224.

41. Hakim MA, Talukder MJ: An assessment of health status of street children in Tangail, Bangladesh. Science Journal of Public Health 2015 , 4(1):1.

42. BBS: Bangladesh Statistical Pocket Book 2019. Bangladesh Bureau of Statistics 2019.

43. Onwe O, Ezeanosike O, Obu C: Economic cost savings with the use of total body phototherapy for the treatment of severe neonatal jaundice in Nigeria. $J$ Pediatr Neonatal Care 2019, 9(6):168-171.

44. Organization WH: Assisting community health workers in India: Dimagi's CommCare. In.: World Health Organization; 2013.

45. Edwards Kl, Itzhak P: Estimated Date of Delivery (EDD). 2019.

46. Kaplan M, Hammerman C: American Academy of Pediatrics guidelines for detecting neonatal hyperbilirubinaemia and preventing kemicterus. Archives of Disease in Childhood-Fetal and Neonatal Edition 2005, 90(6):F448-F449.

47. Group YICSS: Clinical signs that predict severe illness in children under age 2 months: a multicentre study. The Lancet 2008, 371(9607):135-142.

48. Weber MW, Carlin JB, Gatchalian S, Lehmann D, Muhe L, Mulholland EK, Group WYIS: Predictors of neonatal sepsis in developing countries. The Pediatric infectious disease journal 2003, 22(8):711-717.

49. Landis J, Koch G: Landis amd Koch1977_agreement of categorical data. Biometrics 1977, 33(1):159-174.

50. Hategeka C, Ruton H, Law MR: Effect of a community health worker mHealth monitoring system on uptake of matemal and newborn health services in Rwanda. Global health research and policy 2019, 4(1):1-11.

51. Mazumder S, Taneja S, Bahl R, Mohan P, Strand TA, Sommerfelt H, Kirkwood BR, Goyal N, Van Den Hombergh H, Martines J: Effect of implementation of Integrated Management of Neonatal and Childhood Illness programme on treatment seeking practices for morbidities in infants: cluster randomised trial. BMJ 2014, 349 .

Page $12 / 14$ 
52. McConnell M, Ettenger A, Rothschild CW, Muigai F, Cohen J: Can a community health worker administered postnatal checklist increase health-seeking behaviors and knowledge?: evidence from a randomized trial with a private maternity facility in Kiambu County, Kenya. BMC pregnancy and childbirth 2016, 16(1):1-19.

53. Seth A, Tomar S, Singh K, Chandurkar D, Chakraverty A, Dey A, Das AK, Hay K, Saggurti N, Boyce S: Differential effects of community health worker visits across social and economic groups in Uttar Pradesh, India: a link between social inequities and health disparities. International journal for equity in health 2017, 16(1):1-9.

54. Biswas AB, Mukhopadhyay DK, Mandal NK, Panja TK, Sinha N, Mitra K: Skill of frontline workers implementing integrated management of neonatal and childhood illness: experience from a district of West Bengal, India. Journal of tropical pediatrics 2011, 57(5):352-356.

55. Hatzenbuehler L, Zaidi AK, Sundar S, Sultana S, Abbasi F, Rizvi A, Darmstadt G: Validity of neonatal jaundice evaluation by primary health-care workers and physicians in Karachi, Pakistan. Journal of perinatology 2010, 30(9):616-621.

56. Gill CJ, MacLeod WB, Phiri-Mazala G, Guerina NG, Mirochnick M, Knapp AB, Hamer DH: Can traditional birth attendants be trained to accurately identify septic infants, initiate antibiotics, and refer in a rural African setting? Global Health: Science and Practice 2014, 2(3):318-327.

57. Mir SE, Van Der Geest BA, Been JV: Management of neonatal jaundice in low-and lower-middle-income countries. BMJ paediatrics open 2019, 3(1).

\section{Figures}

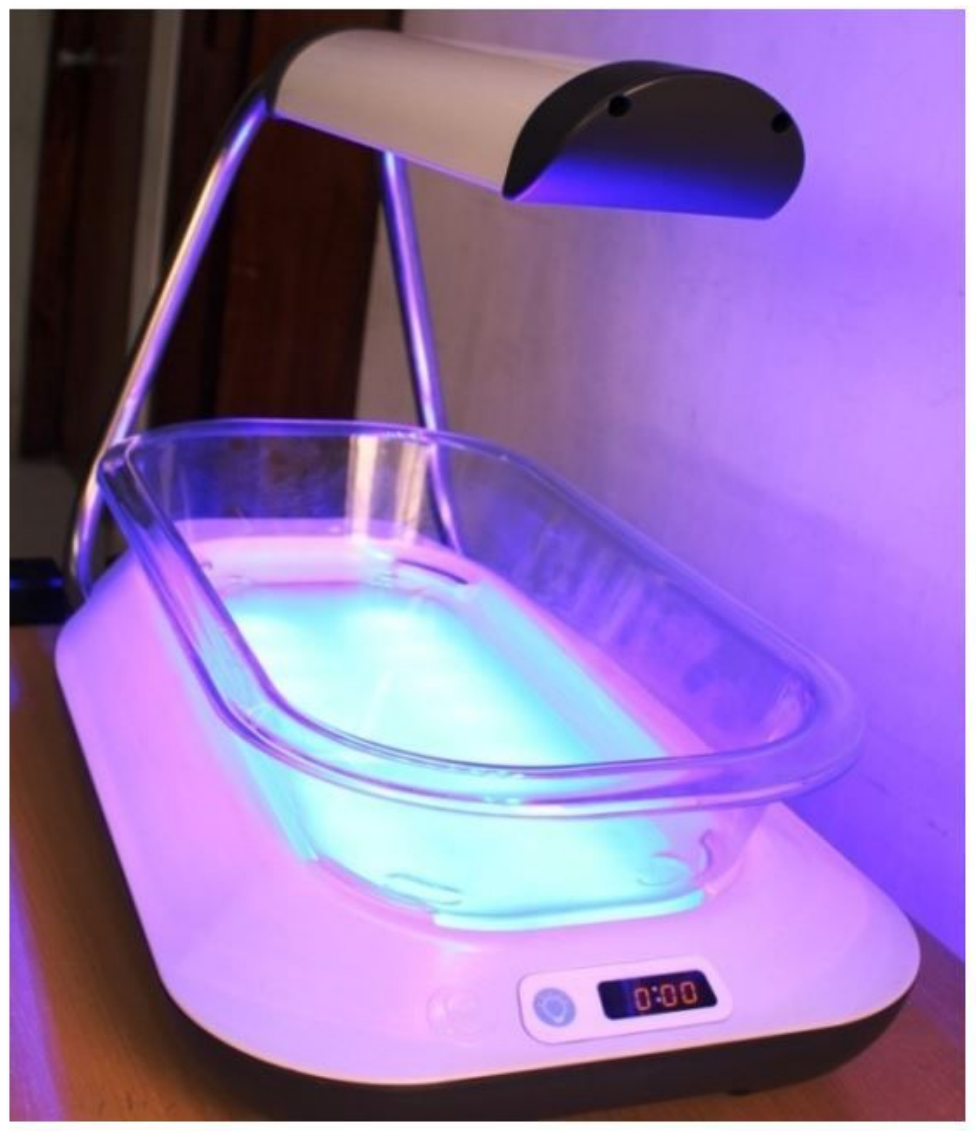

Figure 1

Image of Firefly® phototherapy system (MTTS Asia, Hanoi Vietnam) used in the study (source: icddr,b) 
$<$ 丩 >

নবজাতকেন্ত বিপদের লক্কশতলিত্র মূল्याग़ন

q1. শিশুর কি খ্ঘিচিনি হয়েছে?

ए एों

ना

q2. তাপমাত্রা রেকর্ড করুন (সংখ্যা লিখুন)

98.5

q3. শিশুর বুকের খাঁচ দেবে যাচ্ছে কি না চেক করুন

इ्याँ

নा

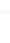

q4. শ্বাসের হার পরিমাপ করুন (সংখ্যা লিখুন)

32

q5. শিশুর নাভির লালচে ভাব পেটেরপ্বকে প্রসারিত হয়েছে কিনা চেক করুল

इ्यों

- ना

Assessment of the newborn danger signs

Has the newborn had history of a convulsion?

Yes

No

Record temperature (In number)

Check if the newborn has severe chest in-drawing Yes

No

Measure the rate of breathing (In number)

Check if the baby has umbilical redness extending to abdominal skin

Yes

No

\section{Figure 2}

Illustration of the mHealth CommCare platform with newborn danger sign assessment (Source: icddr,b) 